\title{
Study on Space-Time Fractional Nonlinear Biological Equation in Radial Symmetry
}

\author{
Yanqin Liu ${ }^{1,2}$ \\ ${ }^{1}$ Department of Mathematics, Dezhou University, Dezhou 253023, China \\ ${ }^{2}$ Nonlinear Dynamics and Chaos Group, School of Management, Tianjin University, Tianjin 30072, China \\ Correspondence should be addressed to Yanqin Liu; yqlin8801@yahoo.cn
}

Received 1 September 2012; Revised 24 December 2012; Accepted 25 December 2012

Academic Editor: Clara Ionescu

Copyright (c) 2013 Yanqin Liu. This is an open access article distributed under the Creative Commons Attribution License, which permits unrestricted use, distribution, and reproduction in any medium, provided the original work is properly cited.

\begin{abstract}
We consider the initial stage of space-time fractional generalized biological equation in radial symmetry. Dimensionless multiorder fractional nonlinear equation was first given, and approximate solutions were derived in the form of series using the homotopy perturbation method with a new modification. And the influence of fractional derivative is also discussed.
\end{abstract}

\section{Introduction}

The problem of biological diffusion is an issue of increasing significance in contemporary ecology $[1,2]$. In case of favourable environmental conditions, the alien population may begin to grow and spread over the area and thus the local initial structural perturbation of the native biological community may lead to large-scale dramatic changes in the community structure. Recently, it has turned out that many phenomena in engineering, physics, chemistry, and other sciences [3-5] can be described very successfully by models using mathematical tools fractional calculus $[6,7]$, such as anomalous transport in disordered systems [8-10], some percolations in porous media, and the diffusion biological population. Mathematical aspects of the biological problem have been considered in many papers [11-13]. El-Sayed et al. [14] studied the fractional-order biological population model in the form $\partial^{\alpha} u / \partial t^{\alpha}=\partial^{2}\left(u^{2}\right) / \partial x^{2}+\partial^{2}\left(u^{2}\right) / \partial y^{2}+f(u)$ using the Adomian decomposition method. Wazwaz and Gorguis [15] gave a detailed study of integer Fisher's diffusion equation by using Adomian decomposition method. Najeeb et al. [16] studied the time fractional Fisher's equation and approximate analytical solutions were obtained by using homotopy analysis method. Petrovskii et al. [17] obtained an exact solution of the spatiotemporal dynamics of a predator-prey community by using an approximate change of variables, and the properties of the solution exhibit biologically reasonable dependence on the parameter values. Liu and Xin [18] studied the fractional Lotka-Volterra equations using the homotopy perturbation method.

This paper is devoted to investigating approximate solutions of a generalized fractional nonlinear population diffusion equation in radical symmetry. The structure of the paper is as follows. In Section 2, a brief review of the theory of fractional calculus will be given to fix notation and provide a convenient reference. In Section 3, a mathematical formulation of the generalized multifractional population diffusion model in radical symmetry is given. In Section 4, we extend the homotopy perturbation method and a new reliable modification to the fractional nonlinear population diffusion system and give some properties of this model. Conclusions and prospects will be presented in Section 5 .

\section{Fractional Calculus}

There are several approaches to define the fractional calculus; the Riemann-Liouville and Caputo fractional operators are defined as follows.

Definition 1. The Riemann-Liouville fractional integral operator $J^{\alpha}(\alpha \geq 0)$ of a function $f(t)$ is defined as

$$
J^{\alpha} f(t)=\frac{1}{\Gamma(\alpha)} \int_{0}^{t}(t-\tau)^{\alpha-1} f(\tau) d \tau, \quad(\alpha \geq 0)
$$


where $\Gamma(\cdot)$ is the well-known gamma function, and some properties of the operator $J^{\alpha}$ are as follows:

$$
\begin{gathered}
J^{\alpha} J^{\beta} f(t)=J^{\alpha+\beta} f(t), \quad(\alpha \geq 0, \beta \geq 0), \\
J^{\alpha} t^{\gamma}=\frac{\Gamma(1+\gamma)}{\Gamma(1+\gamma+\alpha)} t^{\alpha+\gamma}, \quad(\gamma \geq-1) .
\end{gathered}
$$

Definition 2. The Caputo fractional derivative $D^{\alpha}$ of a function $f(t)$ is defined as

$$
\begin{aligned}
& { }_{0} D_{t}^{\alpha} f(t) \\
& =\frac{1}{\Gamma(n-\alpha)} \int_{0}^{t} \frac{f^{(n)}(t) d \tau}{(t-\tau)^{\alpha+1-n}}, \quad(n-1<\operatorname{Re}(\alpha) \leq n, n \in N) .
\end{aligned}
$$

The following are two basic properties of the Caputo fractional derivative:

$$
\begin{gathered}
{ }_{0} D_{t}^{\alpha} t^{\beta}=\frac{\Gamma(1+\beta)}{\Gamma(1+\beta-\alpha)} t^{\beta-\alpha}, \\
{ }_{0} D_{t}^{\alpha}\left({ }_{0} D_{t}^{m} f(t)\right) \\
={ }_{0} D_{t}^{\alpha+m} f(t), \quad(m=0,1,2, \ldots ; n-1<\alpha \leq n), \\
\left(J^{\alpha} D^{\alpha}\right) f(t)=f(t)-\sum_{k=0}^{n-1} f^{(k)}\left(0^{+}\right) \frac{t^{k}}{k !} .
\end{gathered}
$$

We have chosen the Caputo fractional derivative because it allows traditional initial and boundary conditions to be included in the formulation of the problem. And some other properties of fractional derivative can be found in $[3,5]$.

\section{Main Equations}

It is widely accepted that the spatiotemporal dynamics of a biological community can be qualitatively described by diffusion-reaction equations $[2,19]$. A remarkable point is that, in some cases, relatively simple single-species models provide not only qualitative but also quantitative descriptions of the dynamics of a population. In this paper, we first consider a single-species parabolic nonlinear equation arising in the spatial diffusion of biological populations $[11,14]$

$$
\frac{\partial u(x, y, t)}{\partial t}=D\left(\frac{\partial^{2}}{\partial x^{2}}\left(u^{2}\right)+\frac{\partial^{2} u}{\partial y^{2}}\left(u^{2}\right)\right)+f(u),
$$

where $u(x, y, t) \geq 0$ is the population density at position $x, y$, and time $t$, coefficient $D$ describes the intensity of mixing due to animals self-motion, the term $f(u)$ describes multiplication and mortality of a given population, $f(0)=$ $f(K)=0$, and parameter $K$ is being treated as the carrying capacity for the given population. Let us assume that the initial distribution of the species is symmetrical, with the density of the species depending only on the distance from the origin. Assuming also that the environment is homogeneous, that is, both $D$ and $f(u)$ not depends explicitly on the position in space, we arrive at the following problem:

$$
\begin{aligned}
& \frac{\partial u(r, t)}{\partial t} \\
& =D\left(\frac{\partial^{2}}{\partial r^{2}}\left(u^{2}\right)+\frac{1}{r} \frac{\partial}{\partial r}\left(u^{2}\right)\right)+f(u), \quad u(r, 0)=\varphi(r, l),
\end{aligned}
$$

where $0<r<l$ and $l$ is the typical size of the domain and initial condition $\varphi(r, l)$ promptly approaches zero when $r / l \gg 1$. It has turned out that the diffusion of biological population can be described very successfully by fractional calculus. In this paper, we discuss the corresponding fractional equation and the main aim is to solve the nonlinear fractional biological population model in the following form:

$$
\begin{aligned}
\frac{\partial^{\alpha} u(r, t)}{\partial t^{\alpha}}= & D\left(\frac{\partial^{1+\beta}}{\partial r^{1+\beta}}\left(u^{2}\right)+\frac{1}{r} \frac{\partial^{\beta}}{\partial r^{\beta}}\left(u^{2}\right)\right) \\
& +f(u), \quad u(r, 0)=\varphi(r, l),
\end{aligned}
$$

where $0<\alpha \leq 1,0<\beta \leq 1$ is the Caputo derivative. A proper choice of the dimensionless variables, that is, in our case, the choice of scales for the variables $u, r$, and $t$, is an important point. Coming with the property ${ }_{0} D_{t}^{\alpha} f(t)=$ $a_{0}^{-\alpha} D_{t}^{\alpha} f(a t)$ of the Caputo derivative and using reduced dimensionless variables defined as

$$
\tilde{u}=\frac{u}{K}, \quad \tilde{r}=\frac{r}{l}, \quad \tilde{t}=\left(\frac{K D}{l^{1+\beta}}\right)^{1 / \alpha} t
$$

(8) can be reduced to the respective dimensionless forms (tildes will be omitted hereafter):

$$
\begin{aligned}
\frac{\partial^{\alpha} u}{\partial t^{\alpha}}= & \frac{\partial^{1+\beta}}{\partial r^{1+\beta}}\left(u^{2}\right) \\
& +\frac{1}{r} \frac{\partial^{\beta}}{\partial r^{\beta}}\left(u^{2}\right)+F(u), \quad u(r, 0)=\varphi(r, 1),
\end{aligned}
$$

where $F(u)=\left(l^{1+\beta} / K D\right) f(u)$.

\section{Approximate Solution to the Equation}

We now proceed to derive approximate solution to fractional nonlinear population diffusion equation (9).

Case 1. In this case, we will examine the following time-space fractional nonlinear population model:

$$
\begin{gathered}
\frac{\partial^{\alpha} u}{\partial t^{\alpha}}=\frac{\partial^{1+\beta}}{\partial r^{1+\beta}}\left(u^{2}\right)+\frac{1}{r} \frac{\partial^{\beta}}{\partial r^{\beta}}\left(u^{2}\right)+h u, \\
u(r, 0)=r,
\end{gathered}
$$

where $F(u)=h u, h=$ constant, corresponding to the Malthusian law. According to the homotopy perturbation method, we construct the following simple homotopy:

$$
\frac{\partial^{\alpha} u}{\partial t^{\alpha}}=p\left[\frac{\partial^{1+\beta}}{\partial r^{1+\beta}}\left(u^{2}\right)+\frac{1}{r} \frac{\partial^{\beta}}{\partial r^{\beta}}\left(u^{2}\right)+h u\right],
$$


where $p \in[0,1]$ is an embedding parameter. In case $p=0$, (12) is a fractional differential equation, $D_{t}^{\alpha} u=0$, which is easy to solve, and when $p=1,(12)$ turns out to be the original one (10). The basic assumption is that the solutions can be written as a power series in $p$ :

$$
u=\sum_{n=0}^{\infty} p^{n} u_{n}=u_{0}+p u_{1}+p^{2} u_{2}+p^{3} u_{3}+\cdots
$$

$u_{0}$ is an initial approximation of (10). The approximate solutions of the original equations can be obtained by setting $p=1$, that is,

$$
u(x, t)=\lim _{p \rightarrow 1} \sum_{n=0}^{\infty} p^{n} u_{n}=u_{0}+u_{1}+u_{2}+u_{3}+\cdots
$$

Instituting (13) into (12) and comparing coefficients of terms with identical powers of $p$ then applying $J^{\alpha}$ on both sides of equations yield

$$
\begin{gathered}
u_{0}=r, \\
u_{1}=J^{\beta}\left[\frac{\partial^{1+\beta}}{\partial r^{1+\beta}}\left(u_{0}^{2}\right)+\frac{1}{r} \frac{\partial^{\beta}}{\partial r^{\beta}}\left(u_{0}^{2}\right)+h u_{0}\right] \\
=\frac{h r t^{\alpha}}{\Gamma(1+\alpha)}+\frac{2 r^{1-\beta} t^{\alpha}}{\Gamma(1+\alpha) \Gamma(2-\beta)} \\
u_{2}=J^{\beta}\left[\frac{2 r^{1-\beta} t^{\alpha}}{\partial r^{1+\beta}}\left(2 u_{0} u_{1}\right)+\frac{1}{r} \frac{\partial^{\beta}}{\partial r^{\beta}}\left(2 u_{0} u_{1}\right)+h u_{1}\right] \\
=\frac{h^{2} r t^{2 \alpha}}{\Gamma(1+2 \alpha)}+\frac{8 r^{1-2 \beta} t^{2 \alpha}}{\Gamma(1+2 \alpha) \Gamma(2-2 \beta)} \\
\left.+\frac{4 r^{1+\beta}}{\Gamma\left(2 u_{0} u_{2}+u_{1}^{2}\right)+\frac{1}{r} \frac{\partial^{\beta}}{\partial r^{\beta}}\left(2 u_{0} u_{2}+u_{1}^{2}\right)+h u_{2} \beta}\right] \\
+\frac{4 r^{1-2 \beta} t^{2 \alpha} \beta \Gamma(2-\beta)}{\Gamma(1+2 \alpha) \Gamma(2-2 \beta)}+\frac{4 r^{1-2 \beta} t^{2 \alpha}}{\Gamma(1+2 \alpha) \Gamma(3-2 \beta)} \\
+\frac{6 h r^{1-\beta} t^{2 \alpha}}{\Gamma\left(1+2 \beta t^{2 \alpha} \Gamma(2-\beta)\right.}+\frac{6 h r^{1-\beta} t^{2 \alpha}}{\Gamma(1+2 \alpha) \Gamma(3-\beta)}
\end{gathered}
$$

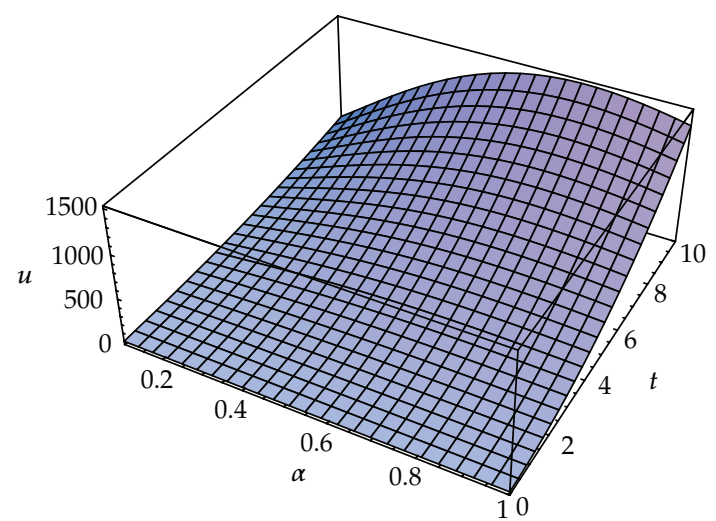

FIGURE 1: The surface of second-order approximate solution of (10) when $r=0.6, \beta=1$.

and so on, in this manner the rest of components of the solution can be obtained. The solution of (10) in series form is given by

$$
\begin{aligned}
u(r, t)= & r+\frac{h r t^{\alpha}}{\Gamma(1+\alpha)}+\frac{2 r^{1-\beta} t^{\alpha}}{\Gamma(1+\alpha) \Gamma(2-\beta)} \\
& +\frac{2 r^{1-\beta} t^{\alpha}}{\Gamma(1+\alpha) \Gamma(3-\beta)}+\cdots
\end{aligned}
$$

Figure 1 shows the approximate solution for (10) and (11) by using the homotopy perturbation method when choosing $r=0.6, \beta=1$. From the figure, it is clear to see the time evolution of nonlinear population diffusion density and we also know that the approximate solution of fractional population model is continuous with the fractional parameter $\alpha$. Figure 2 shows the approximate solution for (10) and (11) when $r=0.6, \alpha=1$, and the approximate solution of fractional population model is continuous with the fractional parameter $\beta$. Figures 3 and 4 show the approximate solution for (10) and (11) when the time $t=10$, from the figures, we also know that the population density changes with the parameters $\alpha, \beta$, and $r$.

Case 2. In this case, we will examine the following time-space fractional nonlinear population model:

$$
\begin{gathered}
\frac{\partial^{\alpha} u}{\partial t^{\alpha}}=\frac{\partial^{1+\beta}}{\partial r^{1+\beta}}\left(u^{2}\right)+\frac{1}{r} \frac{\partial^{\beta}}{\partial r^{\beta}}\left(u^{2}\right)+h u(1-g u) \\
u(r, 0)=e^{r}
\end{gathered}
$$

$F(u)=h u(1-g u), h, g$ are constant, corresponding to the Verhulst law. According to the homotopy perturbation method, we construct the following simple homotopy:

$$
\frac{\partial^{\alpha} u}{\partial t^{\alpha}}=p\left[\frac{\partial^{1+\beta}}{\partial r^{1+\beta}}\left(u^{2}\right)+\frac{1}{r} \frac{\partial^{\beta}}{\partial r^{\beta}}\left(u^{2}\right)+h u(1-g u)\right] .
$$

For this case, it is difficult to solve the multifractional equation. We give a new modification of the homotopy perturbation method; the modified form of the homotopy 


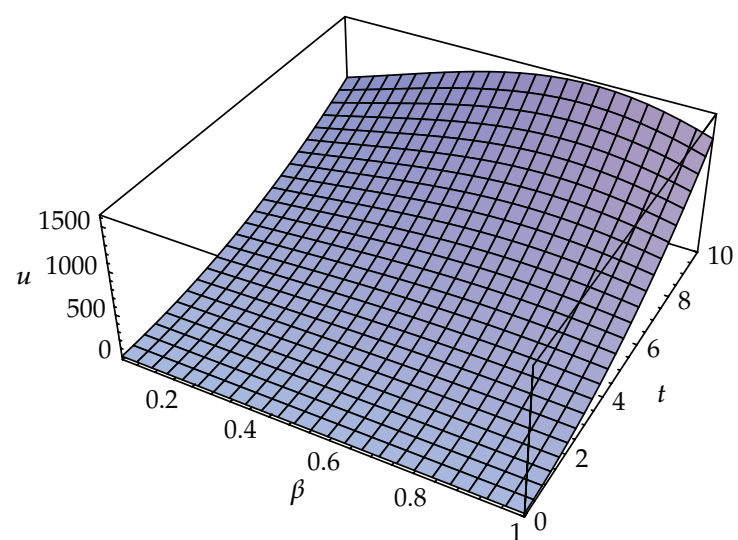

FIgURE 2: The surface of second-order approximate solution of (10) when $r=0.6, \alpha=1$.

perturbation method can be established based on the initial condition expressed in the Taylor series. We suggest that $u(r, 0)$ be expressed in the Taylor series

$$
e^{r}=1+r+\frac{r^{2}}{2}+\frac{r^{3}}{6}+\cdots .
$$

Instituting (13) into (19) and comparing coefficients of terms with identical powers of $p$ then applying $J^{\alpha}$ on both sides of equations yield

$$
\begin{aligned}
& u_{0}=1 \text {, } \\
& u_{1}=r+J^{\beta}\left[\frac{\partial^{1+\beta}}{\partial r^{1+\beta}}\left(u_{0}^{2}\right)+\frac{1}{r} \frac{\partial^{\beta}}{\partial r^{\beta}}\left(u_{0}^{2}\right)+h u_{0}-h g u_{0}^{2}\right] \\
& =r+\frac{(h-h g) t^{\alpha}}{\Gamma(1+\alpha)} \\
& u_{2}=\frac{r^{2}}{2}+J^{\beta}\left[\frac{\partial^{1+\beta}}{\partial r^{1+\beta}}\left(2 u_{0} u_{1}\right)\right. \\
& \left.+\frac{1}{r} \frac{\partial^{\beta}}{\partial r^{\beta}}\left(2 u_{0} u_{1}\right)+h u_{1}-2 h g u_{0} u_{1}\right] \\
& =\frac{r^{2}}{2}+\frac{(h r-2 g h r) t^{\alpha}}{\Gamma(1+\alpha)}+\frac{h^{2} t^{\alpha}}{\Gamma(1+2 \alpha)} \\
& -\frac{3 g h^{2} t^{2 \alpha}}{\Gamma(1+2 \alpha)}+\frac{2 g^{2} h^{2} t^{2 \alpha}}{\Gamma(1+2 \alpha)}+\frac{2 r^{-\beta} t^{\alpha}}{\Gamma(2-\beta) \Gamma(1+\alpha)}, \\
& u_{3}=J^{\beta}\left[\frac{\partial^{1+\beta}}{\partial r^{1+\beta}}\left(2 u_{0} u_{2}+u_{1}^{2}\right)+\frac{1}{r} \frac{\partial^{\beta}}{\partial r^{\beta}}\right. \\
& \left.\times\left(2 u_{0} u_{2}+u_{1}^{2}\right)+h u_{2}-h g\left(2 u_{0} u_{2}+u_{1}^{2}\right)\right] \\
& =\frac{r^{3}}{6}+\frac{h r t^{\alpha}}{\Gamma(1+\alpha)}-\frac{2 h g r^{2} t^{\alpha}}{\Gamma(1+\alpha)} \\
& -\frac{h^{3} g t^{3 \alpha} \Gamma(1+2 \alpha)}{\Gamma^{2}(1+\alpha) \Gamma(1+3 \alpha)}+\frac{2 h^{3} g^{2} t^{3 \alpha} \Gamma(1+2 \alpha)}{\Gamma^{2}(1+\alpha) \Gamma(1+3 \alpha)}
\end{aligned}
$$

$$
\begin{aligned}
& -\frac{h^{3} g^{3} t^{3 \alpha} \Gamma(1+2 \alpha)}{\Gamma^{2}(1+\alpha) \Gamma(1+3 \alpha)}+\frac{h^{2} t^{2 \alpha}}{\Gamma(1+2 \alpha)} \\
& -\frac{g h^{2} t^{2 \alpha}}{\Gamma(1+2 \alpha)}-\frac{4 h^{2} g r t^{2 \alpha}}{\Gamma(1+2 \alpha)}+\frac{6 g^{2} h^{2} t^{2 \alpha}}{\Gamma(1+2 \alpha)} \\
& -\frac{2 h^{3} g t^{3 \alpha}}{\Gamma(1+3 \alpha)}+\frac{6 g^{2} h^{3} t^{3 \alpha}}{\Gamma(1+3 \alpha)} \\
& -\frac{4 g^{3} h^{3} t^{3 \alpha}}{\Gamma(1+3 \alpha)}+\frac{4 r^{1-\beta} t^{\alpha}}{\Gamma(2-\beta) \Gamma(1+\alpha)} \\
& +\frac{4 h r^{-\beta} t^{2 \alpha}}{\Gamma(2-\beta) \Gamma(1+2 \alpha)} \\
& -\frac{6 g h r^{-\beta} t^{2 \alpha}}{\Gamma(2-\beta) \Gamma(1+2 \alpha)}-\frac{4 h g r^{-\beta} t^{2 \alpha}}{\Gamma(2-\beta) \Gamma(1+2 \alpha)} \\
& +\frac{4 r^{1-2 \beta} t^{2 \alpha} \Gamma(1-\beta)}{\Gamma(1-2 \beta) \Gamma(2-\beta) \Gamma(1+2 \alpha)} \\
& +\frac{4 r^{1-\beta} t^{\alpha}}{\Gamma(3-\beta) \Gamma(1+\alpha)}-\frac{4 r^{-1-2 \beta} t^{2 \alpha} \beta \Gamma(-\beta)}{\Gamma(1+2 \alpha) \Gamma(2-\beta) \Gamma(-2 \beta)}
\end{aligned}
$$

and so on, in this manner the rest of components of the solution can be obtained. The solution of (17) in series form is given by

$$
\begin{aligned}
u(r, t)= & e^{r}+\frac{(h-h g) t^{\alpha}}{\Gamma(1+\alpha)}+\frac{(h r-2 g h r) t^{\alpha}}{\Gamma(1+\alpha)} \\
& +\frac{h^{2} t^{\alpha}}{\Gamma(1+2 \alpha)}-\frac{3 g h^{2} t^{2 \alpha}}{\Gamma(1+2 \alpha)}+\cdots
\end{aligned}
$$

Case 3. We will consider the following initial value problem of time-space fractional nonlinear diffusion equation:

$$
\begin{gathered}
\frac{\partial^{\alpha} u}{\partial t^{\alpha}}=\frac{\partial^{1+\beta}}{\partial r^{1+\beta}}\left(u^{2}\right)+\frac{1}{r} \frac{\partial^{\beta}}{\partial r^{\beta}}\left(u^{2}\right)+F(u), \\
u(r, 0)=\varphi(r) .
\end{gathered}
$$

According to the homotopy perturbation method, we construct the following simple homotopy:

$$
\frac{\partial^{\alpha} u}{\partial t^{\alpha}}=p\left[\frac{\partial^{1+\beta}}{\partial r^{1+\beta}}\left(u^{2}\right)+\frac{1}{r} \frac{\partial^{\beta}}{\partial r^{\beta}}\left(u^{2}\right)+F(u)\right] .
$$

The modified form of the homotopy perturbation method can be established based on the initial condition expressed in the Taylor series; the initial condition $u(r, 0)$ is expressed in the Taylor series

$$
u(r, 0)=\varphi(r)=\sum_{n=0}^{\infty} \varphi_{n}(r)
$$




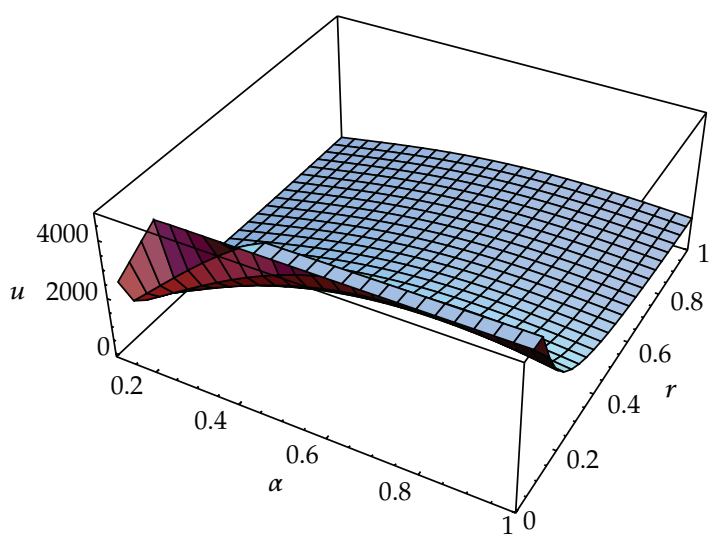

FIGURE 3: The surface of second-order approximate solution of (10) when $t=10, \beta=1$.

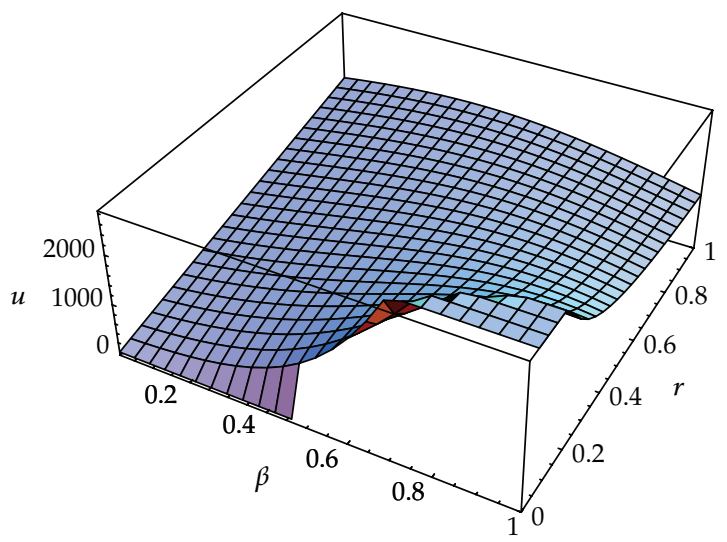

FIGURE 4: The surface of second-order approximate solution of (10) when $t=10, \alpha=1$.

Instituting (12) into (23) and equating coefficients of terms with identical powers of $p$

$$
\begin{gathered}
p^{0}: D_{t}^{\alpha} u_{0}=0, \quad u_{0}(r, 0)=\varphi_{0}(r), \\
\vdots \\
p^{n}: D_{t}^{\alpha} u_{n}=\frac{\partial^{1+\beta}}{\partial r^{1+\beta}}\left(v_{n-1}\right)+\frac{1}{r} \frac{\partial^{\beta}}{\partial r^{\beta}}\left(v_{n-1}\right)+F_{n-1}, \\
u_{n}(r, 0)=\varphi_{n}(r),
\end{gathered}
$$

where $v_{n-1}$ is the coefficient of $p^{n-1}$ in $u^{2}$ and $F_{n-1}$ is the coefficient of $p^{n-1}$ in $F(u)$, then applying $J^{\alpha}$, the inverse operator of $D_{t}^{\alpha}$, on both sides of equations, it is obvious that (27) are easy to solve, the components $u_{n}, n \geq 0$ of the homotopy perturbation method can be completely determined, and series solutions are thus entirely determined.

\section{Conclusion}

Approximate solutions of the multifractional nonlinear diffusion population equations in radial symmetry were derived using the homotopy perturbation method and the new modification of homotopy perturbation method. The solutions are given in the form of series with easily computable terms. The results reveal that the new modified method is very effective for solving nonlinear diffusion equation of multifractional order. This is the first step to study the multifractional nonlinear population diffusion in radical symmetry, and we will make subsequent research, for example, exact solution and self-similar exact solution of these fractional nonlinear system. And we hope that this work is a step in this direction.

\section{Acknowledgments}

The author expresses thanks to the referees for their fruitful advices and comments. This work was supported by the National Science Foundation of Shandong Province (Grants no. Y2007A06 and ZR2010Al019) and the China Postdoctoral Science Foundation (Grant no. 20100470783.)

\section{References}

[1] N. Shigesada and K. Kawasaki, Biological Invasions: Theory and Practice, Oxford University, Oxford, UK, 1997.

[2] S. Petrovskii and N. Shigesada, "Some exact solutions of a generalized Fisher equation related to the problem of biological invasion," Mathematical Biosciences, vol. 172, no. 2, pp. 73-94, 2001.

[3] I. Podlubny, Fractional Differential Equations, Academic Press, New York, NY, USA, 1999.

[4] J. Sabatier, O. P. Agrawal, and J. A. T. Machado, Advances in Fractional Calculus: Theoretical Developments and Applications in Physics and Engineering, Springer, Dordrecht, The Netherlands, 2007.

[5] R. Hilfer, Applications of Fractional Calculus in Physics, World Scientific, Singapore, 2000.

[6] J. T. Machado, V. Kiryakova, and F. Mainardi, "Recent history of fractional calculus," Communications in Nonlinear Science and Numerical Simulation, vol. 16, no. 3, pp. 1140-1153, 2011.

[7] E. K. Lenzi, G. A. Mendes, R. S. Mendes, L. R. Da Silva, and . L. S. Lucena, "Exact solutions to nonlinear nonautonomous spacefractional diffusion equations with absorption," Physical Review E, vol. 67, no. 51, Article ID 051109, 2003.

[8] E. K. Lenzi, L. C. Malacarne, R. S. Mendes, and I. T. Pedron, "Anomalous diffsion, nonlinear fractional Fokker-Planck equation and solutions," Physica A, vol. 319, pp. 245-252, 2003.

[9] M. Bologna, C. Tsallis, and P. Grigolini, "Anomalous diffusion associated with nonlinear fractional derivative Fokker-Plancklike equation: exact time-dependent solutions," Physical Review E, vol. 62, no. 2, pp. 2213-2218, 2000.

[10] C. Tsallis and E. K. Lenzi, "Anomalous diffusion: nonlinear fractional Fokker-Planck equation," Chemical Physics, vol. 284, pp. 341-347, 2002.

[11] F. Shakeri and M. Dehghan, "Numerical solution of a biological population model using He's variational iteration method," Computers \& Mathematics with Applications, vol. 54, no. 7-8, pp. 1197-1209, 2007.

[12] Y. Tan, H. Xu, and S.-J. Liao, "Explicit series solution of travelling waves with a front of Fisher equation," Chaos, Solitons and Fractals, vol. 31, no. 2, pp. 462-472, 2007. 
[13] A. Kadem and D. Baleanu, "Homotopy perturbation method for the coupled fractional Lotka-Volterra equations," Romanian Journal of Physics, vol. 56, no. 3-4, pp. 332-338, 2011.

[14] A. M. A. El-Sayed, S. Z. Rida, and A. A. M. Arafa, "Exact solutions of fractional-order biological population model," Communications in Theoretical Physics, vol. 52, no. 6, pp. 992996, 2009.

[15] A.-M. Wazwaz and A. Gorguis, "An analytic study of Fisher's equation by using Adomian decomposition method," Applied Mathematics and Computation, vol. 154, no. 3, pp. 609-620, 2004.

[16] A. K. Najeeb, K. Nasir-Uddin, A. Asmat, and J. Muhammad, "Approximate analytical solutions of fractional reactiondiffusion equations," Journal of King Saud University, vol. 24, no. 2, pp. 111-118, 2012.

[17] S. Petrovskii, H. Malchow, and B.-L. Li, "An exact solution of a diffusive predator-prey system," Proceedings of The Royal Society of London A, vol. 461, no. 2056, pp. 1029-1053, 2005.

[18] Y. Liu and B. Xin, "Numerical solutions of a fractional predatorprey system," Advances in Difference Equations, vol. 2011, Article ID 190475, 11 pages, 2011.

[19] N. A. Khan, M. Ayaz, L. Jin, and A. Yildirim, "On the approximate solutions for the time-fractional reaction-diffusion equation of Fisher type," International Journal of the Physical Science, vol. 6, pp. 2483-2496, 2011. 


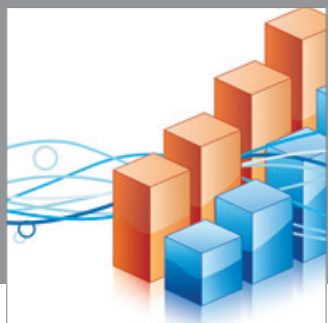

Advances in

Operations Research

mansans

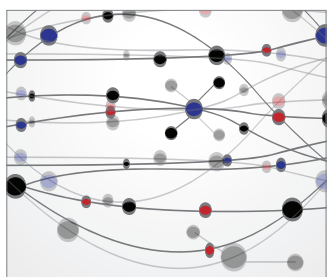

The Scientific World Journal
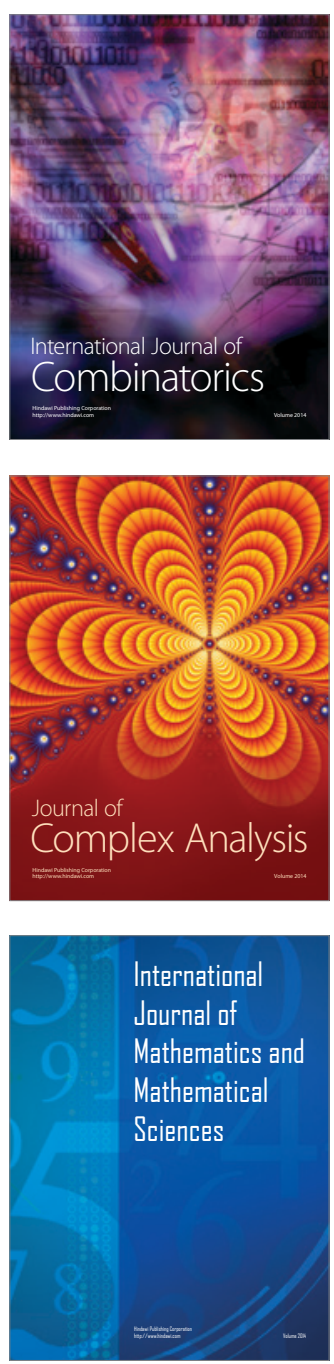
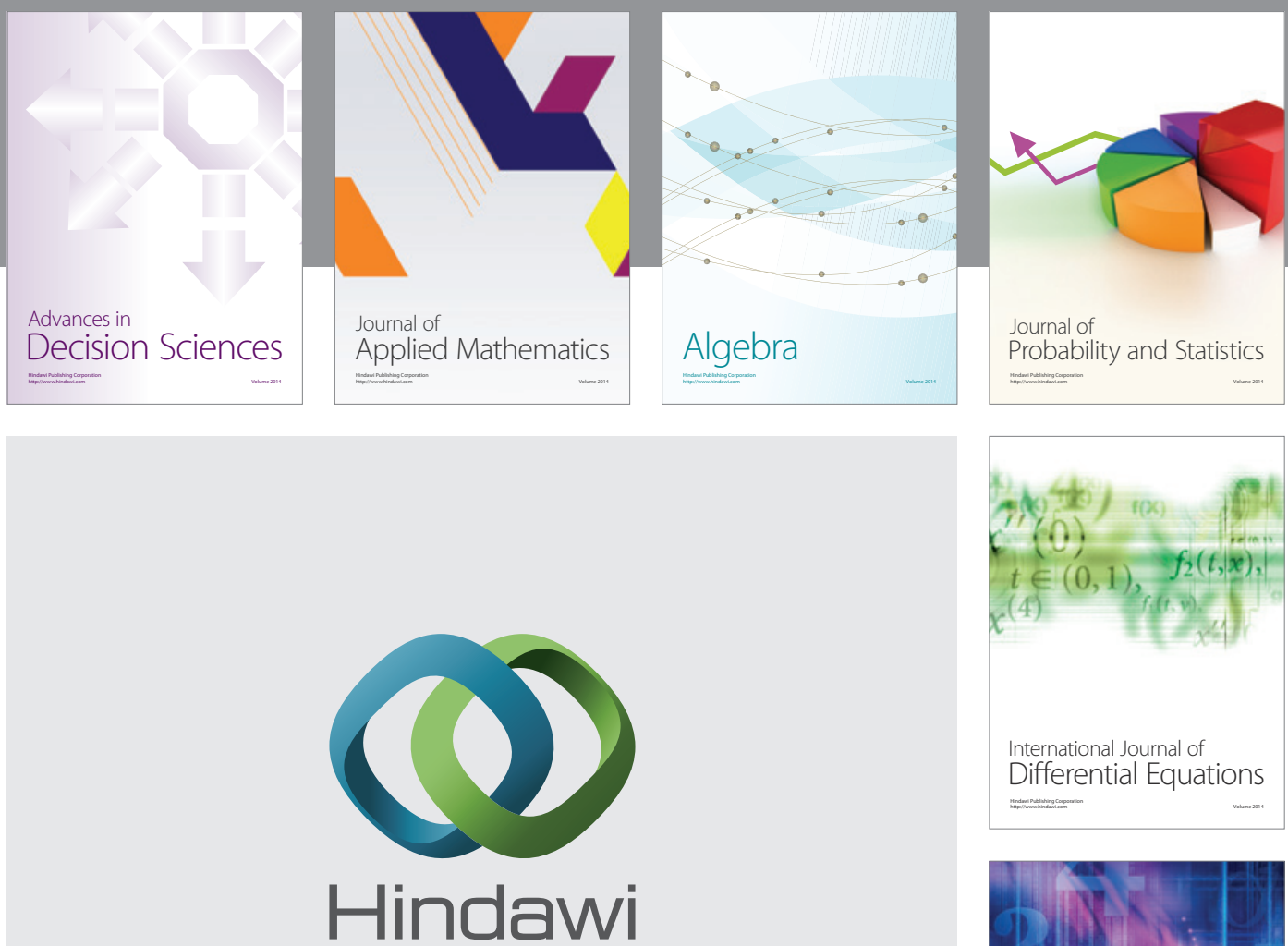

Submit your manuscripts at http://www.hindawi.com
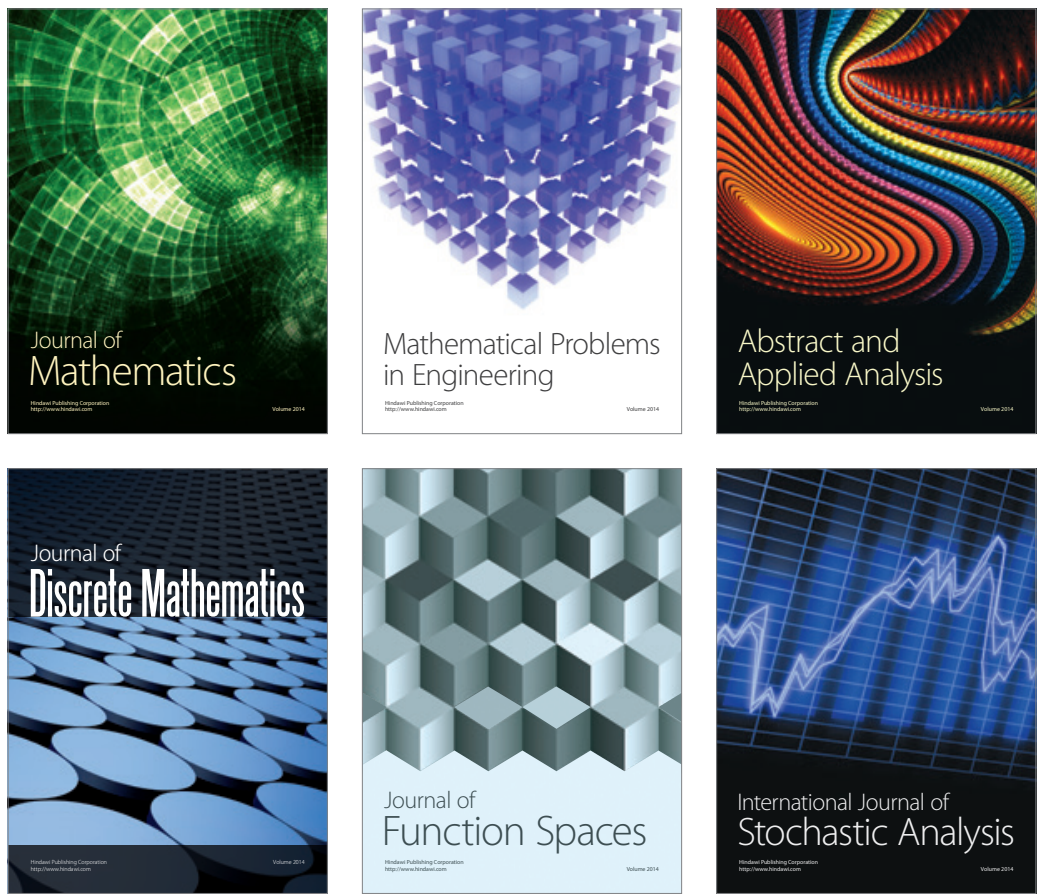

Journal of

Function Spaces

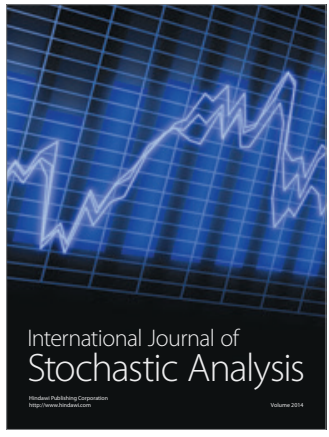

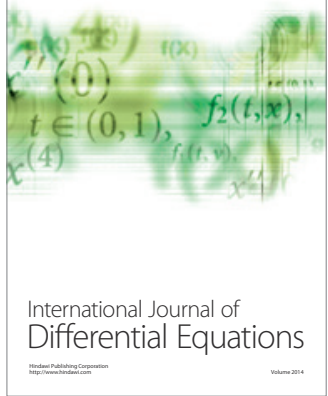
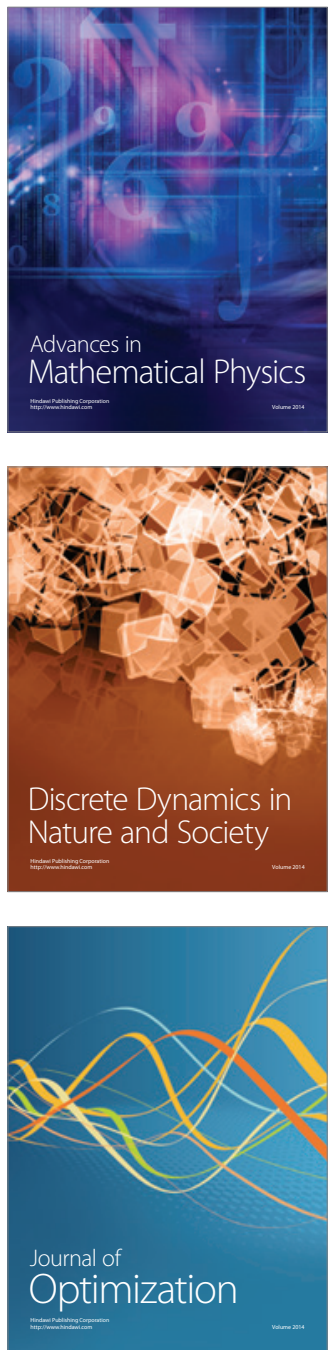